\title{
Numerical Simulation of Strain Localization in Multi-Pass Welded Joints with Account of Residual Stresses
}

\author{
Raisa A. Krektuleva ${ }^{1, \text { a) }}$, Oleg I. Cherepanov ${ }^{2, \text { b) }}$, and Roman O. Cherepanov ${ }^{3, c)}$ \\ ${ }^{I}$ National Research Tomsk Polytechnic University, Tomsk, 654050, Russia \\ ${ }^{2}$ Tomsk State University of Control Systems and Radio Electronics, Tomsk, 634050, Russia \\ ${ }^{3}$ National Research Tomsk State University, Tomsk, 654050, Russia \\ a) Corresponding author: rakrekt@mail.ru \\ b) oi_cherepanov@mail.ru \\ ${ }^{\text {c) }}$ rcherepanov82@gmail.com
}

\begin{abstract}
A two-dimensional elastic-plastic problem was solved to obtain estimation of a residual thermal stresses during cooling of the weld. Quasi-static processes of strain localization in the heat-affected zone were investigated both for compression and tension taking into account influence of residual stress concentrators and heterogeneity of mechanical characteristics of welded materials.
\end{abstract}

Keywords: heat conductivity, multi-pass welding, structural heterogeneity, strain localization, stress concentration, Satoh test

\section{INTRODUCTION}

According to the widely accepted technology, during multi-pass fusion welding, a new layer of weld is normally formed after cooling the previous layers to a temperature of about $500 \mathrm{~K}$. Therefore, the time intervals between the moments of filling weld layers are quite large. The structural heterogeneity and the non-stationary temperature field generate a heterogeneity corresponding to the residual stresses and strain, which influence the strength of the weld. The numerical method proposed in this work allows for modeling a quasi-static process of stress concentration and strain localization during multi-pass welding with account of these features on the basis of a physical mesomechanics approach [1]. The numerical model was developed for 2D modeling, and on this basis stress-strain parameters were calculated that correspond to the experimental data, which confirms the accuracy of the model representations. Model calculations were carried out for the case of V-shaped edge preparation for welding lowalloy steel with a protective layer made of high-alloy steel and metal electrodes with varying degrees of alloying in a five-pass welding process. The numerical model is developed for the simulation of stress concentration and strain localization under the tension, compression and bending of welded joints.

\section{MODEL EQUATIONS}

For calculating the temperature fields and the evolution of the stress-strain state through time, a system of variational equations was used [2,3]. These equations are formulated as follows:

$$
\begin{gathered}
\iiint_{V}\left[\left(\lambda_{i j} d,{ }_{j} T\right) \delta\left(d,{ }_{j} T\right)+C_{\varepsilon} T \delta T\right] \mathrm{d} V^{(n)}-\iint_{S_{G}} n_{i} T \lambda_{i j} \delta\left(d,{ }_{j} T\right) \mathrm{d} S^{(n)}+\iint_{S_{\alpha}} \alpha_{m}\left(T-T_{m}\right)(T \mathrm{~d} t) \mathrm{d} S^{(n)}=0, \\
\iiint_{V}\left(\sigma_{i j}^{E}+\Delta^{*} \sigma_{i j}\right) \delta\left(\Delta^{*} e_{i j}\right) \mathrm{d} V^{(n)}-\iiint_{V}\left(\bar{P}_{i}+\Delta \bar{P}_{i}\right) \delta\left(\Delta u^{i}\right) \mathrm{d} V^{(n)}-\iint_{S_{\sigma}}\left(\bar{R}_{i}+\Delta \bar{R}_{i}\right) \delta\left(\Delta u^{i}\right) \mathrm{d} S^{(n)}=0,
\end{gathered}
$$


where $T$ is the temperature; $\lambda_{i j}$ is the heat conductivity tensor; $\delta\left(d,{ }_{j} T\right)=\left(d,{ }_{j} T\right) \mathrm{d} t$ is the variation of the temperature gradient; $C_{\varepsilon}$ is the specific heat; $\delta T=\dot{T} \mathrm{~d} t$ is the temperature variation; $\alpha_{m}$ is the heat rejection factor; $T_{m}$ is the ambient temperature; $\bar{P}_{i}, \Delta \bar{P}_{i}, \bar{R}_{i}, \Delta \bar{R}_{i}$ are the volume and the surface forces and their increments at the time-step number $(n) ; \Delta u^{i}, \delta\left(\Delta u^{i}\right)$ are the increments of the displacement vector components and their variations; $\Delta \sigma_{i j}^{E}+\Delta^{*} \sigma_{i j}$ is the modified Kirchhoff stress tensor; $\Delta^{*} e_{i j}$ is the modified Green's tensor.

The constitutive equation has the following form:

$$
\Delta^{*} \sigma_{i j}=C_{i j k l}^{*} \Delta^{*} e_{k l}-\beta_{i j}^{*} \Delta T
$$

where $\beta_{i j}^{*}=C_{i j k l}^{*}\left(\alpha_{k l}^{T}+\alpha_{k l}^{F}\right)$, and all the factors are considered as a function of temperature; the tangent modulus tensor $C_{i j k l}^{*}$ was calculated on the basis of equations for an elastic-plastic medium; $\alpha_{k l}^{T}$ are the thermal expansion factors, and the $\alpha_{k l}^{F}$ quantities have the value of the factors of deformation by phase transition.

The replacement of the continuous functions and derivatives in Eqs. (1), (2) with finite differences reduces the problem to solve a system of algebraic equations for nodal temperature $T_{q}^{(n+1)}$ and displacement increments $\Delta u_{q}^{\kappa}$ at each time-step:

$$
\begin{aligned}
& \left\{\left(T_{q}^{(n+1)} \Delta,_{i q}\right) \lambda_{i j}\left(\Delta,{ }_{j p}\right)+\frac{C_{\varepsilon} T_{p}^{(n+1)}}{4 \Delta t}\right\} \Delta V_{p}^{(n)}-\left\{\frac{C_{\varepsilon} T_{p}^{(n)}}{4 \Delta t}\right\} \Delta V_{p}^{(n)}+\alpha_{m}\left(T_{p}^{(n+1)}-T_{m}^{(n+1)}\right)_{p} \Delta S_{p}^{(n)}=0, \\
& \left\{\Delta u_{q}^{\kappa} \frac{\delta_{k l q}^{\kappa}}{2} C_{i j k l}^{*} \frac{\delta_{i j p}^{\beta}}{2}+\left(\sigma_{i j}^{E}-\beta_{i j}^{*} \Delta T\right) \frac{\delta_{i j p}^{\beta}}{2}\right\} \Delta V_{p}^{(n)}-\left(\bar{P}_{p}^{\beta}+\Delta \bar{P}_{p}^{\beta}\right) \Delta V_{p}^{(n)}-\left(\bar{R}_{p}^{\beta}+\Delta \bar{R}_{p}^{\beta}\right) \Delta S_{p}^{(n)}=0,
\end{aligned}
$$

where $\Delta_{i_{i q}}$ are the finite-difference operators to calculate the spatial derivatives of the temperature at the point of the meshwork; $T_{q}^{(n)}$ are the nodal temperatures from the previous time-step, $\Delta u_{q}^{\kappa}$ are increments of the components of the displacement vector; $p$ is the number of mesh cells; $q$ is the number of vertices in the cell; $\delta_{k l p}^{\kappa}$ are the finitedifference operators to calculate the strain tensor components; $\Delta V_{p}^{(n)}, \Delta S_{p}^{(n)}$ are the areas of the surface and length of the sides of the cell at the actual time-step, respectively.
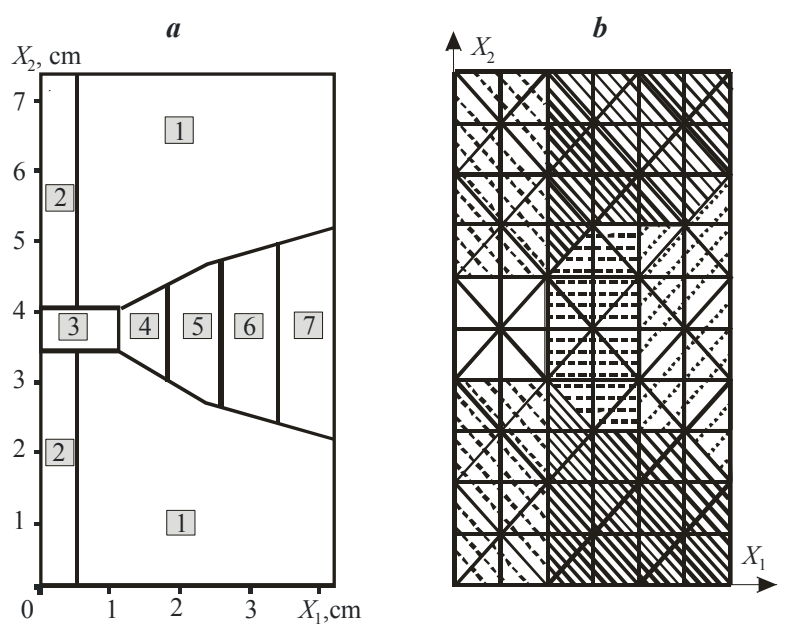

FIGURE 1. Scheme of welded joint: (a) protective layer (1), base metal (2), layers subsequently filled during the multi-pass weld (3-7); (b) scheme of the mesh used for describing the differences of the physical and mechanical characteristics of the elements of the internal structure 
a

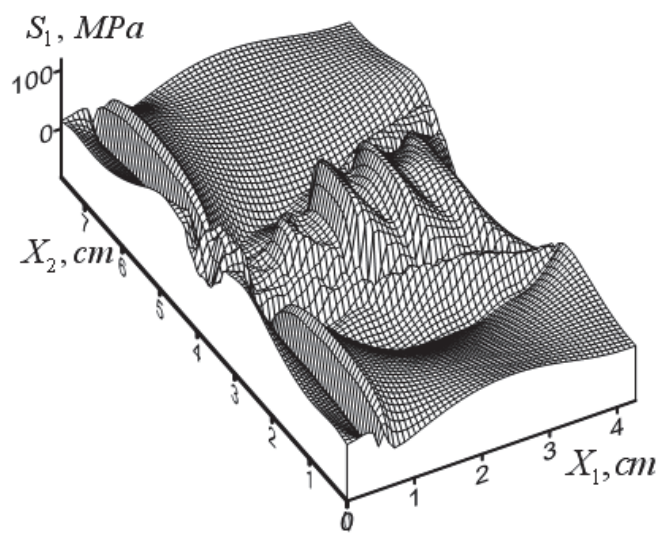

b

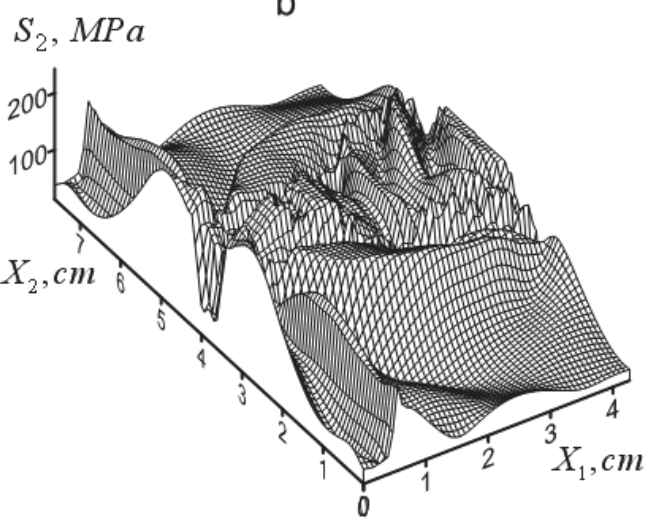

FIGURE 2. Cubic (a) and equivalent (b) residual stresses in the weld after cooling (the case of plane stress)

\section{CALCULATION RESULTS}

We examined the model of a sample with the thickness of the base metal (09G2C steel) of $L_{1}^{1}=4.34 \mathrm{~cm}$ and the thickness of the coating metal $\left(08 \mathrm{Cr} 18 \mathrm{Ni10Ti}\right.$ steel) of $L_{1}^{2}=0.5 \mathrm{~cm}$ with the length of $L_{2}=7.7 \mathrm{~cm}$. The process was simulated for the case of sequential filling of weld with molten metal during five passes of the electrode. Layer 3 is filled at the initial moment (Fig. 1(a)) and then subsequently filled are layers 4-7.

The time delay between the overlay of layers was $4 \mathrm{~s}$, which corresponds to the time characteristic of welding small specimens. One of the features of the described solution is modeling the variation of the temperature field and the stress-strain state in the specimen whose geometry is altered during filling the weld with the melted metal of the electrode. Air was chosen as the external environment for generating the boundary conditions necessary for solving the heat transfer equations, both at the initial moment and before filling is performed; areas 4-7 also are considered as the zones which are filled with air.
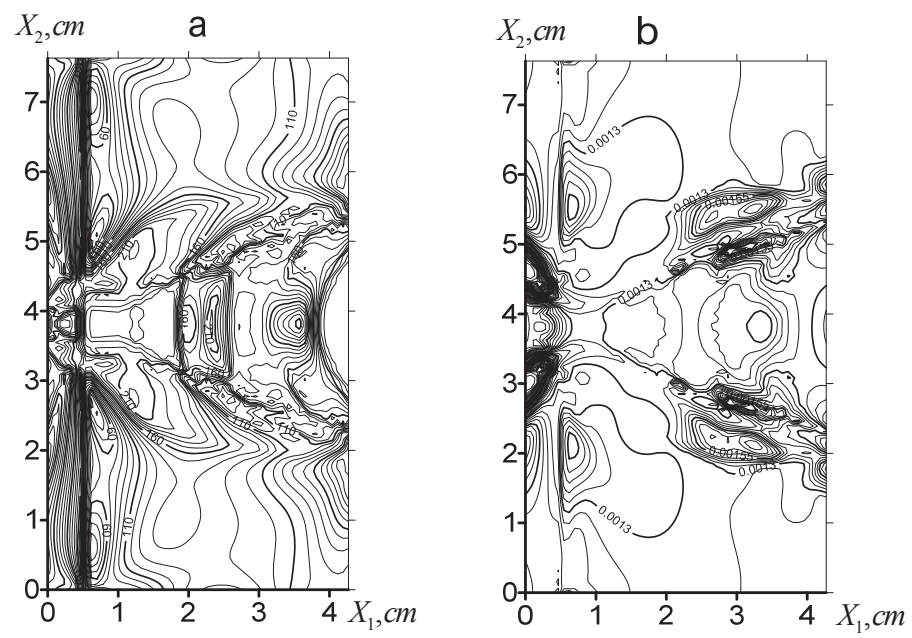

FIGURE 3. Equivalent stress (a) and strain intensity (b) in the modeled sample under tension (mean elongation strain $\varepsilon=1 \cdot 10^{-3}$ ) that were calculated taking into account the residual stresses 

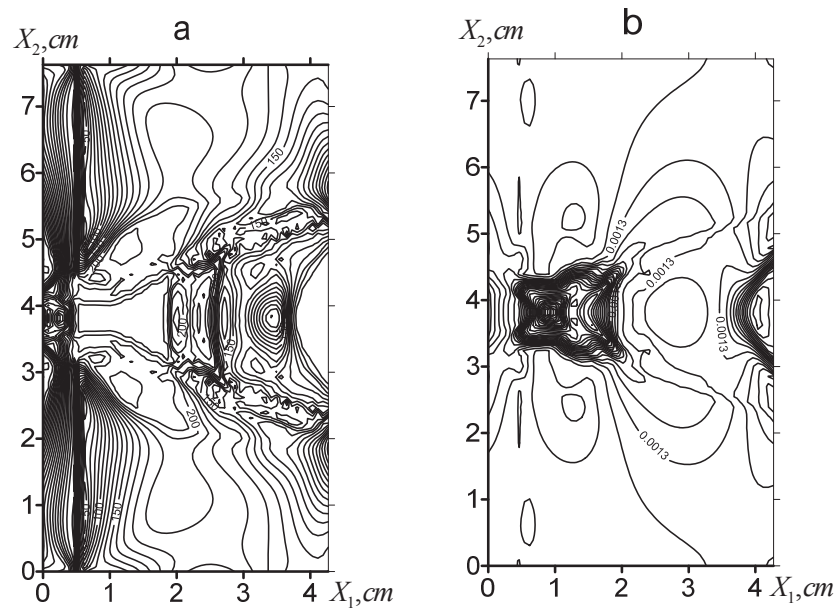

FIGURE 4. Contour map of the equivalent stress (a) and strain intensity (b) of the modeled sample under compression (mean strain $\varepsilon=-1 \cdot 10^{-3}$ ) that were calculated taking into account the residual stresses

One of the features of the proposed calculation method is the use of a mesh with triangular cells shown in Fig. 1(b). In particular, the use of triangular cells allows for improving (to some extent) the accuracy of the description of the structural elements' boundaries as compared to rectangular meshes.

The stress concentration occurs during the solidification of the weld material at all internal borders in the contact zone of the base material and that of the protection layer as well as at the edge borders of the basic material and the material in the weld pool. Figure 2 shows the distribution of residual cubic and equivalent stresses within the completely formed weld after cooling.

The residual stress has influenced the processes of strain localization at subsequent stretching of the modeled sample, especially at the initial stages of elongation. Figure 3 shows the distribution of the equivalent stress and the increments of the strain intensity at the initial steps under stretching when the average elongation is $\varepsilon=\Delta L_{2} / L_{2}=1 \cdot 10^{-3}$ and the residual thermal stresses in the weld were taken into account in the calculation. Under uniaxial compression, the deformations are localized in other areas of the welded joint as compared to elongation (shown in Fig. 4).

For verifying the developed model we have also used the experimental data from scientific literature obtained via the Satoh test performed for different welding alloys [4].

\section{CONCLUSION}

The main result of this work is that the algorithm for the numerical solution of two-dimensional problems of heat conductivity and thermoplasticity has been modified for solids with variable geometry in order to investigate the processes of multi-pass welding of dissimilar steels. It is shown that the initial heterogeneity of the structure and the heterogeneity of the temperature fields lead to the formation of the areas where stress concentration and strain localization taking place during cooling can create mechanical prerequisites for the fracture of the weld.

This work was supported by the Russian Foundation for Basic Research under Grant No. 13-08-00092.

\section{REFERENCES}

1. V. E. Panin (Ed.), Physical Mesomechanics of Heterogeneous Media and Computer-aided Design of Materials (Cambridge International Science Publishing, Cambridge, 1998).

2. K. Vashizu, Variational Methods in Elasticity and Plasticity (Pergamon Press, Oxford, 1982).

3. M. A. Biot, Variational Principles in Heat Transfer (Oxford University Press, Oxford, 1970).

4. J. A. Francis, S. Kundu, H. K. D. H. Bhadeshia, H. J. Stone, R. B. Rogge, P. J. Withers, and L. Karlsson, J. Pressure Vessel Technology 131, 1 (2009). 